\title{
Articulación entre la praxis investigativa y la formación en el programa de trabajo social de la Universidad de La Salle ${ }^{12}$
}

\author{
Ana Marcela Bueno ${ }^{3}$ \\ Claudia Patricia Roa Mendoza ${ }^{4}$
}

\section{RESUMEN}

La investigación formativa como escenario de fundamentación epistemológica, teórica y metodológica para la producción de conocimiento en estudiantes de trabajo social, es una apuesta que en la Universidad de La Salle tiene relevancia por la posibilidad de construir diálogos no solamente a nivel de la comunidad académica, sino de ésta con los contextos sociales y sus actores, más aún cuando el contexto colombiano, por su complejidad, así lo requiere. En esta medida, el Programa de Trabajo Social se ha propuesto articular la praxis investigativa y la formación mediante dispositivos comunes que favorecen el sentido de la producción de conocimiento en la disciplina, denominados líneas de investigación intervención. A través de ellas se han logrado consolidar algunos campos de conocimiento en pro de afianzar la misión profesional en un país con persistente vulneración de derechos, altos índices de pobreza y desigualdad, condiciones sociopolíticas marcadas por un largo conflicto armado, entre otros problemas. Estos

1 Artículo recibido el 15/05/2017. Artículo aprobado el 21/08/2017.

2 Este trabajo sintetiza los principales resultados de: a) seguimiento curricular al Plan de estudios 2008, construido sobre el eje transversal de las líneas de investigación, como campos de conocimiento del trabajo social, b) de la tesis doctoral "Desafíos a la formación profesional en ciencias sociales desde las problemáticas sociales coyunturales, en el Doctorado en Educación y Sociedad de la Universidad de La Salle, línea de investigación "Saber educativo, pedagógico y didáctico" (2015-2018) realizada por Claudia Patricia Roa y de la tesis doctoral "Construcción disciplinar en Trabajo Social: Tendencias en Colombia y Argentina", del Doctorado en Trabajo Social de la Universidad Nacional de Rosario, Argentina, realizada por Ana Marcela Bueno.

3 Colombiana, Trabajadora Social, Universidad Colegio Mayor de Cundinamarca, e-mail: abueno@unisalle.edu.co.

4 Colombiana, Psicóloga, Universidad de La Salle, Bogotá, Colombia, e-mail: claroa@ unisalle.edu.co

4 Las líneas del Programa son: Derechos humanos y fortalecimiento democrático, Familia, realidades, cambios y dinámicas, Perspectivas, debates y prácticas del desarrollo, Sociedad y construcción de subjetividades y Construcción Disciplinar. 
problemas requieren de los aportes de las ciencias sociales y es por ese motivo que las líneas se vislumbran como escenarios de desarrollo del trabajo social en diálogo con la realidad colombiana.

Palabras clave: currículo, líneas de investigación, intervención, investigación formativa.

\section{Articulação entre a práxis de pesquisa e a formação no programa de trabalho social da Universidade de La Salle}

\section{RESUMO}

A pesquisa formativa como cenário de fundamentação teórica e epistemológica, para a produção de conhecimento em estudantes de trabalho social é uma aposta que na Universidade de La Salle, tem relevância pela possibilidade de construir diálogos não só ao nível da comunidade acadêmica, mas também com os contextos sociais e seus atores, mais ainda quando o contexto colombiano, devido à sua complexidade, assim se requer. Nesse sentido, o Programa de Trabalho Social se propus articular a práxis de pesquisa e a formação através de dispositivos comuns que favorecem o sentido da produção do conhecimento na disciplina, denominados linhas de pesquisa intervenção. Através delas, lograram-se consolidar alguns campos de conhecimento para fortalecer a missão profissional num país com persistentes violações dos direitos, altos níveis de pobreza e desigualdade, condições sócio-políticas marcadas por um longo conflito armado, entre outros problemas. Estes problemas exigem das contribuições das ciências sociais e é por esta razão que as linhas são vistas como cenários de desenvolvimento do trabalho social em diálogo com a realidade colombiana.

Palavras-chave: currículo, linhas de pesquisa, intervenção, pesquisa formativa.

\section{Articulation between research praxis and training in the social work program of the University of La Salle}

\section{ABSTRACT}

The formative research as a scenario of epistemological, theoretical and methodological foundation for the production of knowledge in social work students is a relevant commitment at University of La Salle for the possibility of creating dialogues not only at the academic community level, but between this and the social contexts and their actors, even more as the Colombian context requires it due to its complexity. To this extent, the Social Work Program has proposed to articulate research praxis and training through common devices favoring the sense of knowledge production in the discipline, called as lines of research intervention ${ }^{1}$. Through them it has been possible to consolidate some fields of knowledge in order to strengthen the professional mission in a country with persistent 
violation of rights, high levels of poverty and inequality and socio-political conditions marked by a long armed conflict, among others. These issues require contributions from the social sciences and for that reason, the lines are seen as development scenarios for social work in dialogue with the Colombian reality.

Keywords: Curriculum, research lines, intervention, formative research

\section{Introducción}

La denominada sociedad del conocimiento demanda a las Universidades el posicionar un lugar a la producción de nuevo conocimiento, de nuevos saberes que aporten al avance de la ciencia, la tecnología y el desarrollo social en la perspectiva de contribuir a la comprensión y solución de problemas sociales. Esto se hace particularmente importante para el caso de los programas ubicados en el campo de las ciencias sociales, ya que es propio de estos el:

Preguntarse por la sociedad y sus fundamentos, indagar cuáles son las realidades, los cambios, las posibilidades de ser y existir en medio de una sociedad que reúne estilos de vida, de pensamiento, de sentir y de actuar diferentes, distantes y en muchos casos excluyentes (Roa, 2016, p. 77).

Para dar respuesta a esta demanda, las Instituciones de Educación Superior (IES), en el cumplimiento de sus funciones sustantivas:

A saber, docencia, investigación y extensión, requieren hoy de formas que les permitan crear vínculos que faciliten su integración no solo con campos disciplinares y profesionales, sino también con entornos sociales con el fin de materializar los desafíos que en la actualidad se imponen a la universidad (Roa, 2016, p. 108).

En este contexto de las IES, la investigación se convirtió en un proceso necesario para su desarrollo y en uno de los campos desde los cuales se determina la calidad, la que es definida mediante una serie de criterios establecidos en Colombia por el Ministerio de Educación Nacional y el Consejo Nacional de Acreditación. 
Este último, en los lineamientos para la acreditación ${ }^{5}$ de programas de pregrado sitúa la investigación como un factor fundamental en el marco del aseguramiento de la calidad, argumentando que:

Un programa de alta calidad, de acuerdo con su naturaleza, se reconoce por la efectividad en sus procesos de formación para la investigación, el espíritu crítico y la creación, y por sus aportes al conocimiento científico, a la innovación y al desarrollo cultural. De ella se desprenden diversos procesos de alta pertinencia para las IES: la construcción (Consejo Nacional de Acreditación, 2013, p. 40).

Es decir, que desde las políticas nacionales existe una preocupación explícita por el fortalecimiento de la investigación en escenarios educativos, ligada a la generación de nuevo conocimiento, la difusión y socialización del mismo y la consolidación de redes académicas de investigación.

La investigación operacionaliza en tres grandes procesos, a) investigación generativa, la que apunta a la generación de nuevo conocimiento y es llevada a cabo por investigadores reconocidos en un campo particular; b) investigación formativa, orientada al fortalecimiento de competencias de los estudiantes universitarios acordes con el perfil de formación y egreso; c) gestión de la investigación, centrada en los procesos de orden administrativo, que son requeridos para el diseño, ejecución y evaluación de las dos anteriores.

$\mathrm{Al}$ articular estos procesos, puede denotarse, tal como indica Restrepo (2002), que "cuando se habla de investigación en este contexto se hace referencia no sólo a hacer investigación, sino también, y por lo menos, a ser capaz de consumir investigación y de utilizarla pertinentemente en la docencia" (p. 1). En este sentido,

5 "El proceso de acreditación de alta calidad supone el cumplimiento de las condiciones previas o básicas de calidad para la oferta y desarrollo de un programa; se refiere fundamentalmente a cómo una institución y sus programas orientan su deber ser hacia un ideal de excelencia, y pueden mostrar alta calidad mediante resultados específicos, tradición consolidada, impacto y reconocimiento social." (Consejo Nacional de Acreditación, 2013, p. 7) 
la función investigativa establece nexos claramente definidos con la docencia y la proyección social -o servicio-. En relación con la docencia, significa poder trasladar sus procesos y resultados al ejercicio de enseñar, y por tanto, a presentar a los estudiantes los nuevos debates teóricos y metodológicos que se han construido alrededor de diversos campos de conocimiento; así como a la contextualización y actualización de las temáticas que hacen parte de un espacio académico. Con respecto al servicio, implica poner en contextos diversos, los resultados de la investigación para aportar con la solución de problemáticas de carácter social.

Esta doble relación otorgada a la investigación, obliga a pensar ¿qué ha pasado con los procesos de investigación formativa en el contexto de la Universidad Colombiana?, asunto de constante preocupación para la comunidad académica del Trabajo Social en este país.

En ese sentido, la formación en investigación como acto pedagógico, se convierte en un proceso que, más allá del ejercicio esquemático de enseñanza-aprendizaje, supone considerar la concepción de país, individuo, ciudadano, y en consecuencia, del modelo de sociedad, que de manera consciente o inconsciente, están implícitos en los proyectos pedagógicos de las escuelas de Trabajo Social y en los actores comprometidos en el acto pedagógico ( $\mathrm{Fa}-$ lla, 2012). A su vez, pasa por la reflexión en torno al lugar que ésta ocupa en un programa académico, y fundamentalmente, en una propuesta curricular. La discusión en torno a si en Pregrado se deben o no formar investigadores, no aplica para Trabajo Social, en tanto se reconoce la importancia de este proceso en el fortalecimiento de los profesionales frente a las nuevas demandas del contexto social mundial. El carácter pedagógico y educativo que se le atribuye entonces a la investigación formativa, conduce a la generación de diversas preguntas basadas en el interés que tiene el programa de Trabajo Social de la Universidad de La Salle con respecto a su propia experiencia de generar procesos de formación en investigación: ¿Hacia dónde se orientan las propuestas de formación en investigación? ¿Cuáles son las competencias que se proponen fortalecer en los estudiantes? ¿Cuáles son los enfoques 
que se derivan de ellas? ¿Cuál es la relación entre investigación generativa y formativa?

\section{Marco conceptual}

Acuñar el concepto de investigación formativa, surge desde la necesidad de establecer la relación entre docencia e investigación, lo que levanta un problema de orden pedagógico, a saber, el papel que puede ocupar la investigación en el aprendizaje tanto de la investigación como de los conocimientos derivados de diversos campos disciplinares y/o problemas sociales. Sin embargo, la discusión va más allá de incorporar en los currículos "asignaturas" o "espacios académicos" para la formación de investigadores; si bien éstos constituyen una manera de traducir las intencionalidades formativas en investigación de las instituciones, lo más importante es reconocer el lugar que ocupa este proceso en la formación de nuevos profesionales.

Sobre la formación investigativa y el perfil profesional se reconoció que la investigación es un eje transversal y fundamental de la formación; así como la necesidad de la formación integral del Trabajo Social a partir de generar procesos inter y transdisciplinarios en la construcción de conocimiento socialmente relevante y pertinente" (Falla, 2012, p. 19).

Y aunque los diferentes programas en Trabajo Social se han preocupado por incluir la investigación como eje transversal, y a su vez, articularlo en su desarrollo profesional (Cifuentes, Bolaños, López, \& Giraldo, 2008), también es claro que aún no se logra una bisagra que permita establecer las capacidades investigativas con las cuales cuenta un/a trabajador/a social, ya que precisamente el énfasis con el que formamos es profesionalizante, dejando de lado el ejercicio investigativo (Falla, 2014).

En este sentido, la pregunta por la investigación formativa entra a ser parte de la reflexión sobre los procesos de enseñanza, aprendizaje y sobre la manera en que la investigación puede contribuir de modo significativo en éstos. Para el caso de la enseñanza, la pregunta se refiere a la forma en que los docentes pueden transformar 
sus propuestas de asignaturas o espacios académicos, a partir de los resultados de las investigaciones que realizan; así como también, a la necesidad de consolidar propuestas que estén a la vanguardia de los desarrollos en el conocimiento de un campo disciplinar específico. En el caso del aprendizaje, se trata de pensar la formación de estudiantes en investigación que no sólo logran apropiarse de los referentes teóricos y metodológicos para entender la investigación, sino que, al mismo tiempo, hacen lecturas críticas de la realidad y aprenden estrategias y herramientas para hacer investigación. A ello se sumaría, que el aprendizaje promueva una forma distinta de acercarse a los nuevos conocimientos y apropiarlos en su formación profesional, de manera que impacten, no solo el proceso de formación individual, sino que reditúen los procesos curriculares y los procesos ligados a investigación generativa.

Rojas (2006) señala que la investigación formativa ha venido siendo considerada como:

Una estrategia de enseñanza y de aprendizaje que puede o no funcionar porque promueve más el sentido reflexivo y problematizador de toda docencia, que la investigación que dicta el método científico en el sentido estricto. La docencia permite plantear problemas, insistir en ellos, ensayar miradas diversas a las cuestiones duras de la ciencia, invitar a otros a la recapitulación del conocimiento disponible y hacerse responsable del discurso académico, sin que ello necesariamente esté vinculado con una formación programada de investigadores (p. 1).

Esto plantea la necesidad de considerar la investigación formativa como un problema de pedagogía y didáctica que conlleva a la implementación de estrategias de enseñanza-aprendizaje de descubrimiento y construcción (Rojas, 2009). Para ello se requieren habilidades de flexibilidad, adaptabilidad e interdisciplinariedad, o por lo menos el espacio para plantear y manejar problemas de una manera abierta e inductiva. En otras palabras, "se trata de formar profesionales capaces de actualizarse permanentemente y que hayan aprendido a aprender" (Hernández, 2003, p. 187). 
Por su parte, Restrepo (2002) indica que la investigación formativa es un:

Tipo de investigación que se hace entre estudiantes y docentes en el proceso de desarrollo del currículo de un programa y que es propio de la dinámica de la relación con el conocimiento que debe existir en todos los procesos académicos, tanto en el aprendizaje por parte de los alumnos, como en la renovación de la práctica pedagógica por parte de los docentes. Es una generación de conocimiento menos estricta, menos formal, menos comprometida con el desarrollo mismo de nuevo conocimiento o de nueva tecnología (p. 7).

En el desarrollo de la investigación formativa caben algunos cuestionamientos relacionados con la relación entre enseñanza e investigación, y los límites de la enseñanza en la investigación. La definición de estas interrogantes surge en una reflexión que Rojas (2006) hace con respecto a esta relación: "la enseñanza y el aprendizaje procuran una relación inteligente entre personas, aunque, como lo expresa Ibáñez, la unidad de comunicación ya no es la persona sino el rol" (Ibáñez, 1994). O más concretamente, la interacción comunicativa y la forma social de presentación de las personas (Patiño-Garzón \& Rojas, 2009), permite entender el papel esencial de la docencia en el acto educativo, pese a la supuesta crisis de la educación tradicional (Botero, 2005) y a la resignificación de la relación docenteestudiante, donde las escenificaciones de la acción educativa procuran el dinamismo entre sujetos, objetos y contextos en un marco de reflexividad y objetivación como dispositivo cultural (Noya, 1995), "para que la enseñanza tenga sentido y esté legitimada en procesos y resultados en la incorporación de elementos estructurales y de acción social" (Patiño-Garzón \& Rojas, 2009, p. 98).

En ese contexto, el concepto de investigación formativa connota la idea de una profunda transformación en la manera de educar a los individuos, especialmente en la modernidad temprana (Riehl, 2001); de hecho la idea de "formar" es una cuestión relativamente nueva en la historia de la educación, cuyo centro ordenador viene dado por el cambio de perspectiva epistemológica, donde la 
existencia del sujeto, concepto dado en la modernidad por la teoría del conocimiento, reemplaza la noción de individuo (Botero, 2005). Lo anterior, haciendo hincapié que "es conveniente para el Trabajo Social, asumir un compromiso no sólo en la acción, como tradicionalmente lo ha hecho, sino con la reflexión y creación de conocimiento" (Falla, 2009, p. 47).

\section{La investigación en la Universidad de La Salle}

La Universidad de La Salle reivindica la investigación como uno de los componentes fundamentales de su identidad, incluyéndola en una de sus funciones: "una Universidad... que, realiza investigación con pertinencia e impacto social..." Así mismo, dentro de su misión y visión se compromete con "la generación de conocimiento que aporte a la transformación social y productiva del país..." (Universidad de La Salle, 2007, p. 7). Todo esto se resume en el lema de la Institución de una "Universidad que aprende porque investiga".

Responsable de su papel como ente académico que debe reflexionar y aportar a los problemas, asume la investigación con impacto social como un proceso articulador de su praxis, en el marco del desarrollo humano integral y sustentable, constituyendo a la investigación formativa en un camino a la investigación científica, fomentándola en los procesos académicos, lo cual debe estar orientado a la motivación por la indagación, la crítica, la generación de pensamiento autónomo y el conocimiento de la realidad (Universidad de La Salle, 2007, p. 15).

En el Enfoque Formativo Lasallista (EFL), se concibe la formación integral como un proceso de construcción del sí mismo... fundado en el ejercicio responsable de generación de conocimiento con el compromiso de transformar la realidad (Universidad de La Salle, 2007, p. 15). Así mismo, se sugiere una reflexión crítica y constructiva como ejercicio del pensamiento, lo cual permita la transformación de agentes, el mejoramiento e innovación de los contextos de enseñanza-aprendizaje y de generar productos significativos de conocimiento (p. 16). 
En el marco del papel formador que tiene la Universidad, se establece que la investigación social es una articulación entre la formación del pensamiento y la gestión profesional (Programa de Trabajo Social, 2004, p. 8). En ese proceso de formación integral, se asume también que la investigación formativa es una estrategia que favorece un ambiente educativo orientado a que los y las estudiantes sean sujetos activos de su formación (p. 11).

Por otro lado, y resaltando la importancia de las didácticas en los ambientes de formación, el EFL proponen didácticas que fomenten la actitud, las competencias y los resultados investigativos; procedimientos relacionados con la lectura analítica, la pedagogía de la pregunta, la pedagogía por descubrimiento, la observación, la sistematización, la interpretación, la capacidad crítica, argumentativa y propositiva (Universidad de La Salle, 2007, p. 8).

\section{La investigación formativa en el Programa de Trabajo Social}

\section{Fundamentación}

El Programa de Trabajo Social contribuye en la formación de profesionales y en la generación de conocimiento en interacción con el contexto cultural, sociopolítico, económico y ambiental. En este sentido, se articulan las funciones de docencia, investigación y proyección social como ámbitos de reflexión y compromiso que permiten concretar los propósitos del programa y aportar en la transformación de las prácticas sociales (Programa de Trabajo Social, 2008).

Para el Programa de Trabajo Social de la Universidad de La Salle, la investigación formativa siempre ha estado presente como parte integral de la formación, la que se sustenta en los siguientes supuestos: a) la relación directa entre la investigación y la calidad de la educación superior; b) la investigación como articuladora entre la docencia y la extensión; c) la investigación como soporte de la intervención, lo cual permite una comprensión crítica de la realidad; d) la investigación como fundamento para la construcción disciplinar y por ende para la consolidación profesional. Todos 
ellos se convirtieron en argumentos que sostienen la intencionalidad prioritaria de la función investigativa para el programa (Camelo \& Ordóñez, 2006).

Conforme a la normatividad legal vigente, se entiende por Trabajo Social la profesión ubicada en el campo de las Ciencias Sociales y Humanas, que interviene en procesos y proyectos relacionados con los derechos humanos, políticas sociales y desarrollo humano y social; tiene como fundamento la democracia, el bien común, justicia y libertad, en el marco de la actividad profesional (Consejo Nacional de Trabajo Social, 2002).

El Ministerio de Educación Nacional incluye a Trabajo Social en el nivel profesional universitario como parte del área de Humanidades y Ciencias Sociales (Ministerio de Educación Nacional, 2007).

En la trayectoria del Trabajo Social a nivel latinoamericano, se considera que en el proceso de formación de los y las trabajadores sociales, debe evidenciase un desarrollo articulado entre la investigación social y los procesos de intervención profesional del Trabajo Social, a partir del desarrollo de líneas y grupos de investigación, y el énfasis en la formación integral de los profesionales, mediante el diseño de estrategias pedagógicas, consistentes en cualificar su desarrollo profesional.

De acuerdo a los lineamientos del CONETS, el/la trabajador/a social se forma como investigador social con el objeto de esclarecer la realidad social para proponer alternativas de intervención pertinentes según el caso. Para ello, los y las estudiantes deben formar competencias que integren el desarrollo del pensamiento lógico; el análisis documental y los estados del arte; la construcción teórica y metodológica de los problemas; entre otros elementos que son fundamentales en el ejercicio investigativo (Consejo Nacional para la Educación en Trabajo Social, 2000).

La investigación en la formación en Trabajo Social es vital en la medida que se trata de un proceso que permite involucrar las dimensiones personal, social y profesional de los estudiantes, ade- 
más de promover las competencias del ser, el saber y el conocer mediante el desarrollo de actividades desarrolladas en contexto, las cuales son fundamentales para su formación integral, pues además de participar de manera activa en los contextos, intercambia experiencias en diversos escenarios sociales (Camelo \& Ordóñez, 2006).

\section{Por tanto}

La configuración del Trabajo Social como profesión o como disciplina ha estado ligada a la formación en investigación, por lo que ha sido objeto de análisis y preocupación no sólo en los diferentes programas académicos, sino también entre docentes y entre los organismos que guardan relación con la profesión (Falla, 2009, p. 51).

Así se asume el reto de realizar una reestructuración de la relación docencia-investigación-proyección social, donde, a partir de las líneas de investigación del programa, se revisaron los espacios académicos presentes en la malla curricular, estableciendo vínculos, de tal forma que cada espacio pertenece a una línea, con el fin de que sean nutridas recíprocamente por las discusiones, reflexiones, y productos derivados de ambos procesos.

El programa de Trabajo Social de la Universidad de La Salle promueve la generación de conocimiento en constante interacción con el contexto cultural, sociopolítico, económico y ambiental del país, en el marco de una formación ética y política de profesionales y postgraduados, la investigación en trabajo social y en otros campos de las ciencias sociales y humanas, la reflexión crítica sobre el contexto social y la política pública y la incidencia en los procesos que promueven los derechos humanos, el desarrollo pleno de las personas y la construcción de una sociedad justa, equitativa y democrática. (Programa de Trabajo Social, 2010).

Lo anterior puede ser sintetizado en el siguiente gráfico donde se intenta plasmar la relación que desde y para el currículo se realiza de docencia-investigación-proyección social. 
Figura 1: Articulación docencia, investigación y proyección social

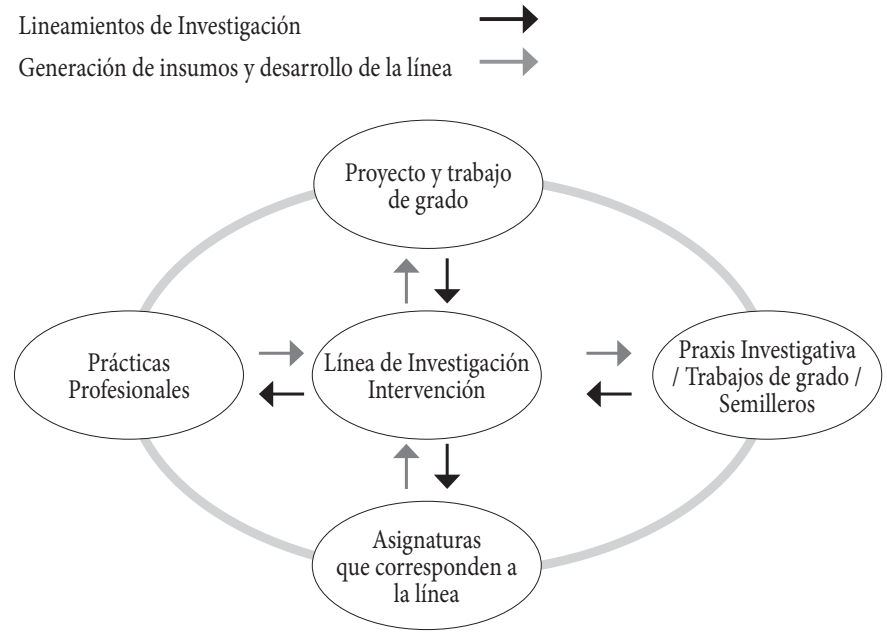

Fuente: Presentación líneas de investigación e intervención, Programa de Trabajo Social 2013

De esta forma, se da un salto trascendental de una formación en investigación ligada al desarrollo de competencias, en unos espacios específicos, a un concepto de investigación que transversaliza la malla curricular, haciendo un currículo vivo que va más allá de las asignaturas y que recibe una retroalimentación constante de la discusión en torno a los núcleos problémicos, investigativos y a las líneas de investigación.

\section{Estrategias}

Uno de los componentes a destacar en el Programa de Trabajo Social de la Universidad de La Salle, es la formación en investigación social, mediante la estrategia de investigación formativa, la cual articula la reflexión epistemológica, teórica y metodológica en la comprensión de los problemas sociales, los sujetos, los métodos para el desarrollo profesional, asumiéndose en el currículo, espacios académicos específicos para el proceso de enseñanza aprendizaje, con proyectos articulados a las líneas y grupos de investigación del programa, la creación y consolidación de semilleros de investigación, y actividades de socialización de los procesos y productos investigativos (Programa de Trabajo Social, 2010). 
Entre las estrategias pedagógicas que utilizan los docentes para fomentar el espíritu investigativo, están la problematización acerca de los fenómenos a través del planteamiento de preguntas y respuestas devueltas para la discusión; construcción de un criterio propio respecto al conocimiento; desarrollo de la investigación como proceso en términos de la aproximación y construcción permanente con respecto al objeto de conocimiento (Ordóñez, 2009).

El proceso formativo en el currículo, se estructura a partir de las áreas curriculares, las cuales plantean un proceso secuencial en la formación, demarcando la articulación entre los procesos de fundamentación, profesionalización y complementariedad. En la malla curricular, la praxis investigativa se configura en un trasversal que atraviesa la formación desde la primera macrocompetencia ${ }^{6}$ con "construcción del conocimiento social", seguido de los espacios de "interpretación de la realidad social" y medición de problemas sociales”, siendo estos espacios teóricos de fundamentación, pero que se unen con espacios prácticos en la segunda macrocompetencia ${ }^{7}$, como lo son: "Diseño de proyectos de investigación social" y "desarrollo de proyectos de investigación social”. A su vez, en el nivel de la cuarta macrocompetencia ${ }^{8}$ aparecen los espacios académicos que le permiten al estudiante desarrollar sus apuestas en este nivel con el "proyecto de grado" y "el trabajo de grado".

La investigación formativa, tal como se plantea en el currículo, es una estrategia didáctica que permite aprender a investigar investigando, y se implementa mediante espacios de interacción teóricoprácticos conformados por subgrupos de estudiantes con la tutoría de un/a docente para vivenciar el proceso de construcción de

6 Se refiere a la capacidad de utilizar los aportes de las ciencias sociales y humanas y la fundamentación del trabajo social para la comprensión de la sociedad, las culturas y los sujetos sociales.

7 Capacidad para identificar y proponer alternativas a las dinámicas sociales en el marco de los derechos humanos y el desarrollo humano integral y sustentable.

8 Capacidad para proponer alternativas interdisciplinarias de cambio social. 
proyectos para acceder al conocimiento, comprensión y análisis de los problemas sociales para su transformación.

Para los espacios de proyecto y trabajo de grado, existen algunas modalidades de grado ${ }^{9}$ que facilitan la flexibilidad en ese sentido, aunque todas ellas requieren del ejercicio de investigación.

Estas modalidades son definidas así:

\section{a. Desarrollo de un proyecto investigativo disciplinar o inter-} disciplinar: Esta modalidad de grado se refiere al desarrollo de proyectos de investigación que responden a problemáticas concretas en diferentes contextos socioeconómicos y culturales. Se fundamenta en la aplicación de los avances disciplinares de los diferentes campos de conocimiento o en la interacción complementaria de diferentes disciplinas para abordar problemáticas sociales del contexto local, regional o nacional (Capítulo II, Artículo 3, Acuerdo No. 002 de 2017, Facultad de Ciencias económicas y sociales, 2017).

b. Participación activa en proyectos de investigación disciplinar o interdisciplinar: Se entiende como la participación de los estudiantes en investigaciones cuyo objeto de estudio se aborda a partir de disciplinas particulares o en proyectos en los cuales la solución al problema de estudio, se asume articuladamente por varias disciplinas. Estos proyectos pueden ser desarrollados por colectivos docentes de la Universidad o de estos en asociación con equipos externos. El rol que los estudiantes cumplen en esta modalidad, puede ser como coinvestigadores, como asistentes de investigación o como miembros activos de un semillero de investigación. En cualquier caso, el estudiante debe participar activamente en el proceso durante mínimo tres períodos académicos consecutivos. (Lineamientos para la

9 Acuerdo No. 002 de 2017 del Consejo de Facultad por el cual se reglamentan las modalidades de grado, de pregrado y maestría de la Facultad de Ciencias Económicas y sociales, en correspondencia con lo establecido en los diseños curriculares. 
gestión curricular de la praxis investigativa. P.83-84) (Artículo 3, Acuerdo 002 de 2017).

c. Producción intelectual relevante: La condición relevante en esta modalidad de grado está dada por la calidad de la producción que se haya generado. En tal sentido, la naturaleza del producto debe cumplir con claros estándares de calidad, innovación, sistematicidad y correspondencia respecto a los propósitos formativos del programa al cual se encuentra vinculado el estudiante. Así, se asumen como producción intelectual relevante la publicación de un libro o capítulo de libro; un artículo publicado en una revista, institucional o externa, especializada en el campo o campos de conocimiento inherentes al programa del estudiante. Estas publicaciones deben contar con los respectivos registros de ISBN o ISSN, dependiendo de si es un libro o si aparecerá en una publicación periódica, bien sea en formato digital o impreso (Artículo 3, Acuerdo 002 de 2017).

d. Co-grado: es una oportunidad académica que afianza la articulación entre pregrado y posgrado, así un estudiante de la Universidad de La Salle puede cursar espacios académicos de especialización o maestría como modalidad de grado, a partir de los créditos previstos en la malla curricular de su programa de origen. En correspondencia con lo establecido en el reglamento para estudiantes de Postgrado, los espacios académicos aprobados como modalidad de grado podrán ser homologados en este nivel de formación (Artículo 3, Acuerdo 002 de 2017).

\section{Líneas de Investigación}

La investigación como eje curricular en el programa se desarrolla en torno a las líneas de investigación e intervención; estas líneas permiten ubicar la praxis investigativa en campos disciplinares e interdisciplinares en los cuales estudiantes y docentes desarrollan proyectos de investigación formativa y de investigación propiamente dicha, realizada por docentes. 
Dichos procesos deben articularse a las líneas de investigación e intervención del Programa, las cuales son consideradas como campos de conocimiento sobre los cuales se puede desarrollar una estructura global de investigación, que se traduce en proyectos alrededor de núcleos temáticos, adecuados a las complejidades del entorno social, político, cultural y regional en que se inscriben, los cuales esperan ser analizados (Facultad de Trabajo Social, 2002). En la actualidad, las líneas de investigación vigentes en el programa son:

- Perspectivas, debates y prácticas del desarrollo: la que centra su interés en una "visión integral del ser humano dentro de criterios éticos, de equidad, sostenibilidad y sustentabilidad; haciendo que la persona fomente sus capacidades a partir de sus potencialidades, para producir creación cultural, solidaridad social, libertad política, oportunidades de empleo e igualdad de género" (Programa de Trabajo Social, 2010).

- Derechos Humanos y Fortalecimiento Democrático: sustentada en el acuerdo histórico que la AIETS y la FITS 2004 han hecho sobre la definición internacional de Trabajo Social, acorde con el mundo globalizado y los problemas y necesidades propias del siglo XXI. Reconoce también que los derechos humanos constituyen parámetros para identificar el grado de desarrollo y el carácter democrático de un país.

- Familias: Realidades, cambios y dinámicas, se encuentra articulada en el campo de producción investigativa y formativa en el tema de familia. Ella se articula a los espacios de formación e investigaciones desarrolladas por los y las estudiantes y las docentes en cuanto que la familia se asume como un objeto de conocimiento e intervención de Trabajo Social.

- Construcción Disciplinar: La justificación de la existencia de esta línea surge ante la necesidad de consolidar la construcción conceptual, como aporte para la formación en las unidades académicas de Trabajo Social a nivel nacional e internacional, y afinar la construcción de preguntas sobre su funda- 
mentación. En esta línea se incluyó como sub línea el desarrollo curricular, la cual se ocupaba de analizar el currículo y los asuntos relacionados con la formación en trabajo social.

- Sociedad y Construcción de Subjetividades: Reconoce al sujeto en interacción con sus diversos entornos, los cuales están atravesados por dimensiones como la cultura, la política, la economía y otros. Permite un análisis de la sociedad y los procesos cotidianos en los que el ser humano construye su subjetividad.

\section{Semilleros de investigación}

Como Programa de Trabajo Social se considera que la formación en investigación, debe ir más allá del trabajo en el aula, motivo por el cual se propuso la estrategia de Semilleros de investigación, la cual está orientada a complementar la formación en investigación que se desarrolla en el currículo, por ende, como procesos de formación conexa, deben articularse a las estructuras institucionales. En este sentido éstos se vinculan a las líneas de investigación e intervención del programa y/o con proyectos de investigación docente. Específicamente, la estrategia de los semilleros se implementa a partir de las siguientes opciones:

- Apoyo al desarrollo de las líneas de investigación, mediante la actualización de los estados del arte, consultas bibliográficas, análisis de temáticas específicas, o incluso, en el desarrollo de habilidades relacionadas con recolección de información, como aplicación de técnicas e instrumentos, sistematización y análisis de información.

- Apoyo a investigación docente: Articulación del aprendizaje en un ejercicio de aprender a investigar, investigando. En esta opción, las/los estudiantes desarrollan las competencias de investigación de la mano de los docentes, quienes ejercen su función como investigadores.

- Desarrollo de proyectos de investigación financiables: Como proceso formador, los semilleros de investigación deben lograr que los estudiantes que complementen su formación con esta 
estrategia, tengan la capacidad de formular proyectos de condiciones posibles para su financiación, en el marco de procesos institucionales que favorecen a los jóvenes investigadores, lo cual no solo permite el desarrollo del proyecto sino la visualización del semillero a nivel externo (p. 56).

Actualmente se encuentran en el programa dos semilleros: CONOCER: Semillero de investigación en Conflicto y Oportunidades de convivencia. Semillero Beatriz Sandoval, pero la estrategia se ha venido afianzando con el correr de los años. Hoy se están gestando dos nuevas apuestas: Salud y Paz y PazSOS.

\section{Conclusiones}

Pasados 8 años del redimensionamiento curricular, los avances de los que se puede hablar hoy día, tienen relación con aquellas articulaciones directas que se han venido construyendo en la relación docencia, investigación y proyección social, pues las líneas, como elementos transversales en el currículo, son las que le permiten a estudiantes, docentes, e incluso administrativos, pensar los horizontes de sentido del programa. Ello puesto que, desde las coordinaciones de línea, se plantean intereses que parten desde los desarrollos investigativos de los docentes, y a su vez, los estudiantes se inscriben de acuerdo a sus expectativas y a las posibilidades de oferta que han surgido en el marco del grupo de investigación, lo cual garantiza que todo confluya en el marco de las líneas.

Si bien la praxis investigativa es un componente transversal en el currículo, es necesario continuar fortaleciéndola con estrategias como los semilleros, que también, desde la articulación curricular, permitan afianzar las destrezas que se requieren alcanzar en la formación como trabajadores sociales investigadores.

Si bien continúa la pregunta por la formación de investigadores, es necesario considerar la investigación como un campo de intervención y no como una etapa de la misma, pues así las cosas, sigue quedando subyugada al hacer, lo cual no permite que se vaya más 
allá de la instrumentalización de la razón (Vélez, 2003). Adicionalmente, porque dentro de las posibilidades de investigación en trabajo social las áreas en las cuales intervenimos son escenarios constantes de construcción de conocimiento, así como el trabajo social mismo, como objeto de desarrollo; por ello, la construcción disciplinar ha sido una línea tradicional en este Programa.

Vale la pena destacar que la investigación formativa en el Programa de Trabajo Social de la Universidad de La Salle, ha cobrado significado desde su nacimiento, pero con el desarrollo de la investigación docente, ésta se logra consolidar cada vez más en pro del reconocimiento de los contextos sociales colombianos y la apuesta por proponer estrategias de intervención acordes a las problemáticas allí identificadas, lo cual es, para el país, un aporte fundamental, especialmente en este momento de transición hacia un camino de paz.

\section{Bibliografía}

Botero, A. (2005). Diagnóstico filosófico de los paradigmas epistémicos y su relación con la universidad latinoamericana. (G. Biogénesis, Ed.) Uni/pluri/versidad, 5(1), 47-58.

Camelo, A. \& Ordóñez, Z. (2006). La investigación formativa en la facultad de Trabajo Social. En C. N. Social, Formación Investigativa en Trabajo Social (págs. 199-207). Cali: Universidad del Valle.

Cifuentes, M. R., Bolaños, N., López, Y. \& Giraldo, C. (2008). Marco de Fundamentación conceptual en Trabajo Social. Bogotá: Consejo Nacional para la Educación en Trabajo Social.

Consejo Nacional de Acreditación (2013). Lineamientos para la acreditación de programas de pregrado. Bogotá: Sistema Nacional de Acreditación.

Consejo Nacional de Trabajo Social (2002). Código de ética profesional. Acuerdo 004. Bogotá.

Consejo Nacional para la Educación en Trabajo Social (2000). Lineamientos básicos para los programas académicos de Trabajo Social en Colombia. Bogotá.

Facultad de Ciencias económicas y sociales (2017). Acuerdo No. 002 de 2017. Bogotá.

Facultad de Trabajo Social (2002). Documento de líneas de investigación. 2002: Universidad de La Salle. 
Falla, U. (julio-diciembre de 2009). Tendencias de la formación investigativa en el trabajo contemporáneo. Revista Trabajo Social (10), 35-64.

Falla, U. ( enero-junio de 2012). La investigación, eje transversal en la formación en trabajo social en Colombia. Espacio Regional, 1(9), 13-27.

Falla, U. (2014). La investigación en el trabajo social contemporáneo. Bogotá: Universidad Colegio Mayor de Cundinamarca.

Hernández, C. (2003). Investigación e investigación formativa. Nómadas (18), 183-193.

Ibáñez, J. (1994). El regreso del sujeto. La investigación social de segundo orden. Madrid: Siglo XXI.

Ministerio de Educación Nacional (2007). Resolución No 466 por la cual se definen las características específicas de calidad para la oferta y desarrollo de programas académicos de formación profesional en Humanidades y Ciencias Sociales. Bogotá.

Noya, F. (1995). Metodología, contexto y reflexividad. Una perspectiva constructivista y contextualista sobre la relación cualitativo-cuantitativa en la investigación social. En J. Delgado, \& J. Gutiérrez Métodos y técnicas cualitativas de investigación en ciencias sociales (págs. 121-140). Madrid: Síntesis.

Ordóñez, Z. (2009). Informe de Autoevaluación con fines de renovación para la Acreditación. Bogotá.

Patiño-Garzón, L. \& Rojas, M. (2009). Subjetividad y subjetivación de las prácticas pedagógicas en la Universidad. Educación y Educadores, 93-105.

Programa de Trabajo Social (2004). Proyecto Pedagógico. Bogotá: Universidad de La Salle.

Programa de Trabajo Social (2008). Proyecto Educativo. Bogotá: Universidad de La Salle.

Programa de Trabajo Social (2010). Currículo Programa de Trabajo Social. En F. d. Sociales, Facultad de Ciencias Económicas y Sociales. Currículos redimensionados (págs. 91-169). Bogotá: Ediciones Unisalle.

Restrepo, B. (2002). Conceptos y aplicaciones de la investigación formativa, y criterios para evaluar la investigación científica en sentido estricto. Bogotá: CNA.

Riehl, C. (2001). Puentes al futuro: las contribuciones de la investigación cualitativa de la sociología de la educación. Albany.

Roa, C. (2016). Desafíos a la formación profesional en ciencias sociales desde las problemáticas sociales coyunturales. Cuadernos 
Articulación entre la praxis investigativa y la formación en el programa de trabajo social de la Universidad de La Salle / Bueno - Roa

de seminario. Reflexiones para una epistemología del saber pedagógico (6), 77-92.

Roa, C. (2016). Investigación en modo 3: Una alternativa para la articulación investigación e intervención en educación superior. Guillermo de Ockham, 14(2), 103-110.

Rojas, M. (2006). La Investigación formativa, sustento y tensión de la docencia en la Universidad. Notas Universitarias, 16, 35-45.

Rojas, M. (2009). La investigación formativa y la docencia en la universidad. Revista Uis Humanidades, 37(2), 107-122.

Universidad de La Salle (2007). Enfoque Formativo Lasallista. Bogotá: Ediciones Unisalle.

Universidad de La Salle (2007). Proyecto Educativo Universitario Lasallista. Bogotá: Ediciones Unisalle.

Vélez, O. L. (2003). Reconfigurando el Trabajo Social. Buenos Aires: Espacio. 